\title{
The Ecdysone receptor constrains wingless expression to pattern cell cycle across the Drosophila wing margin in a cyclin B-dependent manner
}

\author{
Naomi C Mitchell, Jane I Lin, Olga Zaytseva, Nicola Cranna, Amanda Lee and Leonie M Quinn*
}

\begin{abstract}
Background: Ecdysone triggers transcriptional changes via the ecdysone receptor (ECR) to coordinate developmental programs of apoptosis, cell cycle and differentiation. Data suggests EcR affects cell cycle gene expression indirectly and here we identify Wingless as an intermediary factor linking EcR to cell cycle.

Results: We demonstrate EcR patterns cell cycle across the presumptive Drosophila wing margin by constraining wg transcription to modulate $\mathrm{CycB}$ expression, but not the previously identified Wg-targets dMyc or Stg. Furthermore co-knockdown of Wg restores CycB patterning in EcR knockdown clones. Wg is not a direct target of $\mathrm{EcR}$, rather we demonstrate that repression of $\mathrm{Wg}$ by $\mathrm{EcR}$ is likely mediated by direct interaction between the EcR-responsive zinc finger transcription factor $\mathrm{Crol}$ and the wg promoter.
\end{abstract}

Conclusions: Thus we elucidate a critical mechanism potentially connecting ecdysone with patterning signals to ensure correct timing of cell cycle exit and differentiation during margin wing development.

Keywords: Ecdysone, Cyclin B, Drosophila, Cell cycle, Wingless

\section{Background}

Metamorphosis of Drosophila involves proliferation, differentiation and death of larval tissues in order to form the adult fly. The major developmental hormone in Drosophila, the steroid hormone 20-hydroxyecdysone (ecdysone) is secreted from the prothoracic gland (PG) in pulses that precede critical morphological changes during development [1-4]. Ecdysone pulses are required for all aspects of developmental timing and morphogenesis, starting with the formation of the body plan during late embryogenesis required to develop to the first instar larval stage and for the cuticle moulting at the end of the first and second instars. A large titre of ecdysone is released at the end of the third larval instar in preparation for pupation, which marks the beginning of adult tissue metamorphosis [1,2]. Metamorphosis is orchestrated by the cascade of gene transcription triggered by

\footnotetext{
* Correspondence: I.quinn@unimelb.edu.au

* Correspondence: I.quinn@unimelb.edu.au 3010, Melbourne, Australia
}

ecdysone, which activates the ecdysone receptor (EcR), a member of the nuclear receptor family $[1,2]$.

The Drosophila larval wing imaginal disc has long served as an excellent system to elucidate connections between the activity of developmental signals and patterning of cell cycle gene expression, but potential mechanism(s) modulating these events via ecdysone/EcR remain a mystery. The wing disc is comprised of an epithelial sheet, which can be divided into distinct domains based on cell fate in the adult wing; the notum, hinge and pouch (Figure 1A). With the release of the ecdysone hormone at the end of the third instar, proliferation of the wing imaginal disc slows and differentiation of the adult sensory neurons begins along the presumptive wing margin [5,6]. Cell division is tightly coupled with differentiation in the cells comprising the wing margin, which undergo a cell cycle delay in order to pattern proneural gene expression in the clusters of sensory neuron precursor (SOP) cells required for differentiation and development of bristles $[7,8]$. However, a subset of 


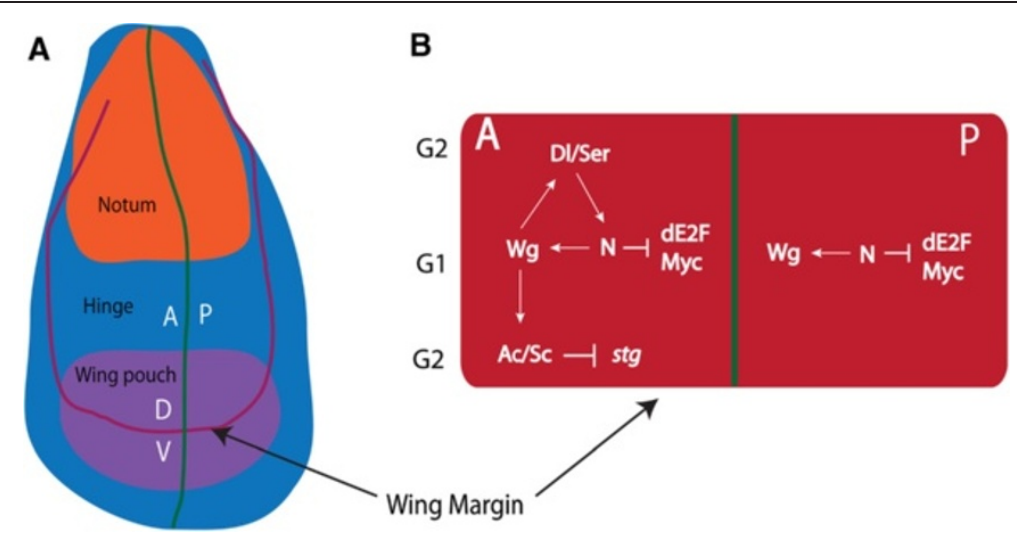

Figure 1 Patterning cell cycle across the wing imaginal disc. (A) - Schematic of the 3rd instar wing disc. The red and blue region develops to form the notum and hinge while the purple region forms the wing blade. The green line marks the anterior-posterior (A/P) boundary while the red line defines the dorsal-ventral (DN) boundary. (B) - Across the margin Notch (N) expression triggers the activation of Wingless (Wg). In the margin Wg induces G1 delay via repression of dE2F1 activity in a narrow domain within the anterior compartment and also in a broader region of the posterior compartment. In the anterior compartment, Wg also induces $\mathrm{G} 2$ delay in the cells flanking the G1 band by down regulating expression of stg.

margin cells must remain competent to re-enter the cycle as bristle precursors do not complete their final cell divisions until 24 hours After Puparium Formation (APF), by which time all epithelial cells of the wing have exited the cell cycle and most cells have arrested in G1 $[5,6]$. Thus for proper timing of wing margin development, cells spanning the dorsal-ventral (D/V) boundary must first undergo a coordinated cell cycle delay, but must also be competent to re-enter the cell cycle to complete bristle cell divisions during early pupal stages.

Interactions between the Wingless (Wg) secreted morphogen and the Notch $(\mathrm{N})$ receptor pathway have been implicated in this cell cycle patterning across the presumptive wing margin [9-12]. Although all cells at the $\mathrm{D} / \mathrm{V}$ boundary are cell cycle delayed during the late third instar, patterning between the anterior and posterior compartments differs; posterior cells both within and immediately flanking the $\mathrm{D} / \mathrm{V}$ boundary are delayed in G1, while the G1 band across the anterior of the boundary is flanked by G2 delayed cells (Figure 1B) [9-11,13,14]. During the transition from second to third instar, the $\mathrm{D} / \mathrm{V}$ boundary is established through the activity of Notch signaling, via the Notch ligands Serrate and Delta, which lead to activation of $w g$ expression [15-17]. Through an autoinhibitory effect, Wg refines its own expression and also promotes expression of Delta and Serrate to create a positive-feedback loop that maintains Notch signaling and restricts Wg expression to the D/V boundary [18-20]. Wg signaling leads to the expression of proneural genes achaete $(a c)$ and scute $(s c)$ specifically within the anterior compartment of the cells flanking the D/V boundary [21], which results in down regulation of Stg and delay of these cells in G2 [9]. The activity of Notch and Wg signaling pathways spatially regulate the activity of bantam micro-
RNA and the expression levels of the dMyc to mediate regulation of the E2F transcription factor and the transition of G1 to S-phase $[10,11]$. Thus the interplay between $\mathrm{Wg}$ and $\mathrm{N}$ is essential for orchestrating the cell cycle exit across the presumptive margin, which is required for sensory neuron differentiation and development of the wing margin.

Microarray analysis has revealed that the ecdysone signal is associated with modulation of cell cycle regulatory pathways such as Wg, Notch and Dpp during mid-gut morphogenesis [22]. Ecdysone/EcR is also critical for coupling growth and proliferation in the abdominal histoblasts [23]. During larval stages histoblasts grow in a G2 arrested state prior to entering a proliferative stage during pupal metamorphosis [24,25] and the transition to a proliferative state is initiated by ecdysone-dependent activation of the essential G2-M phosphatase String/Cdc25 (Stg) [26]. However, as the stg promoter lacks an EcRE, further experiments are required to identify the factors mediating the observed transcriptional activation of stg required for G2-M progression. Ecdysone can also control animal growth rate via EcR-dependent expression of the growth and S-phase regulator dMyc in the fat body [27]. In this system, loss of EcR function in fat body results in elevated $\mathrm{dMyc}$ expression and increased growth, suggesting that EcR signaling normally represses dMyc. However, like the stg promoter, $d m y c$ lacks an EcRE suggesting that the repression of $\mathrm{dMyc}$ is unlikely to occur via direct transcriptional regulation by EcR.

Ecdysone pulses therefore control developmental timing and growth of a range of larval tissues [28-30], but how does ecdysone achieve these changes in cell growth and cell cycle progression? In particular, how does ecdysone connect with the major developmental signaling 
pathways that regulate cell cycle patterning in Drosophila imaginal tissues? Here we demonstrate that the release of ecdysone at the end of the third instar is likely to control the timing of the cell cycle delay and initiation of differentiation across the presumptive wing margin via EcR, which is essential for refining $w g$ expression and for patterning the cell cycle delay. While dMyc and Stg are key targets of the Notch and Wg pathways, we show that $\mathrm{CycB}$ is the major cell cycle target downstream of EcR and Wg. EcR is essential for ensuring $\mathrm{CycB}$ expression is maintained along the presumptive wing margin, but not away from the margin and loss of $\mathrm{CycB}$ expression in the EcR knockdown clones is dependent on the presence of $\mathrm{Wg}$ at the margin.

The $w g$ promoter lacks an EcRE, suggesting $w g$ transcription is unlikely to be directly regulated by EcR. Rather we provide evidence that the effect of EcR on Wg and, therefore, $\mathrm{CycB}$ is mediated by the ecdysone/EcRresponsive zinc finger transcription factor Crol. Expression of crol is sufficient to restore $w g$ repression in the EcR loss of function background, and ChIP revealed that Crol is normally enriched at consensus zinc-finger binding sites within the $w g$ promoter. We have therefore added another arm to the mechanism patterning the cell cycle delay across the presumptive wing margin at the end of third instar, whereby parallel pathways can act on Wg to drive a G2 delay; signaling through EcR/Crol-Wg down regulates $\mathrm{CycB}$ while interaction between Notch and Wg inhibits Stg. Thus we propose that the pulse of ecdysone at the end of third instar normally ensures proper timing of the cell cycle delay across the presumptive wing margin via EcR and the Wg-repressor Crol, which ensures expression of $w g$ is confined to the $\mathrm{D} / \mathrm{V}$ boundary and controls timing of the $\mathrm{G} 2$ delay via $\mathrm{CycB}$.

\section{Results}

\section{EcR is essential for cell cycle patterning throughout the} wing margin

As EcR is abundantly expressed along the wing margin (Figure 2A-D), we hypothesised that the rise of ecdysone levels at the end of the third instar larval period might be required to pattern cell cycles during this critical stage of wing metamorphosis. In wing imaginal discs, DNA synthesis is coupled with cell division; cells grow in G1, initiate DNA replication and enter S-phase, which is separated from mitosis by G2 phase. To first monitor S-phase progression, we used the PCNA-GFP reporter, which gives a read out of E2F1 transcription factor activity and, therefore, indicates whether cells are in late G1 or S-phase [31]. The pattern of E2F1 activity across the apical surface of the wing disc epithelium in a wild type background together with the overlapping pattern of EcR protein is shown in Figure 2 (A-D). PCNA-GFP is normally detected in cycling cells of the wing pouch and in the G1 cells within the anterior and posterior of the wing margin, but decreased in the G2 cells of the anterior margin. EcR protein, detected using the EcR common antibody to both EcR A and B isoforms [32], is ubiquitously expressed throughout the wing pouch, but shows relatively higher levels of expression across the D/V boundary. Thus EcR staining overlaps with PCNA-GFP throughout the pouch and the G1 cells of the margin.

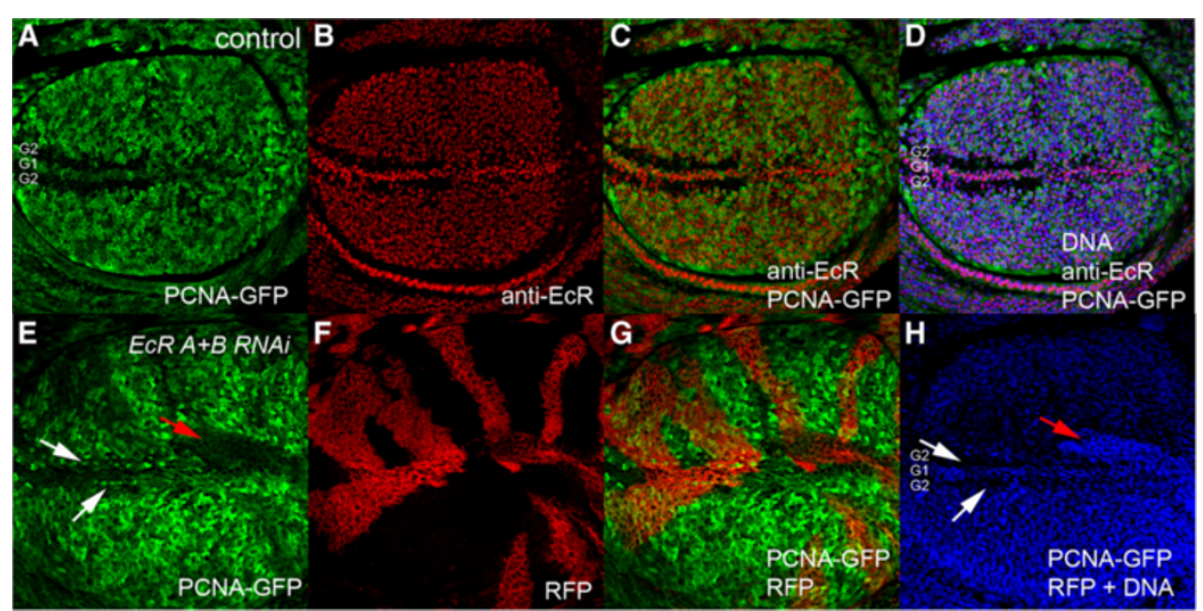

Figure $2 \mathrm{EcR}$ is required for normal patterning of E2F1 activity in the wing margin. (A-D) - PCNA-GFP/+ wing imaginal discs stained with the ECR antibody. PCNA-GFP (A) is normally detected in cycling cells of the wing pouch and in the G1 band within the anterior and posterior of the wing margin. ECR staining in red (B) overlaps with PCNA-GFP throughout the pouch and the G1 cells of the margin (C and $\mathbf{D}$, DNA in blue). (E-H) - ECR RNAi flip out clones generated in the PCNA-GFP background are marked with CD8-RFP. White arrows mark ectopic G1 cells in the G2 region. The DNA stain in $\mathrm{H}$ is for the equivalent confocal section and white arrows show the position of ectopic PCNA-GFP corresponding to clones in the anterior, while the red arrow corresponds to the clone with reduced PCNA-GFP in the posterior. 
To test whether EcR knockdown affected E2F activity we generated flip out clones [33] using the previously characterised $U A S-E c R$ RNAi targeted to both EcRA and EcRB receptors [34]. Across the presumptive wing margin, EcR RNAi disrupted E2F1 patterning, in both the anterior cells flanking the boundary, with GFP detected in many of the cells that should normally be delayed in G2 (Figure 2E, white arrows) and also appeared to decrease PCNA-GFP in the posterior margin (Figure 2E, red arrows). The EcR pathway is, therefore, required for normal patterning of E2F1 transcription factor activity across the margin of the presumptive wing blade.

\section{EcR is required for $\mathrm{Wg}$ repression, but only partially regulates E2F1 activity via $\mathbf{W g}$}

Our previous work revealed a potential link between ecdysone signaling and the Wg pathway, as we demonstrated that the ecdysone-responsive transcription factor Crol [35] is required for repression of $\mathrm{Wg}$ in the third instar wing disc [36]. Given that inhibition of the Wg pathway across the margin has been associated with ectopic activation of cell cycle regulators $d m y c$ and stg, which leads to ectopic cells in S-phase and mitosis $[9,10,13,14]$, we set out to determine whether the disruption to E2F1 activity in EcR loss-of-function clones was mediated by Wg. EcR protein is abundant in the $w g$ expressing cells (marked by $w g$-lac $Z$ ) at the margin, but is also expressed in surrounding non-wg expressing cells throughout the wing imaginal disc (Figure $3 \mathrm{~A}-\mathrm{C}$ ). Consistent with our previous study using the EcR dominant negative transgenes [36], EcR RNAi results in an expansion of $w g$ promoter activity (Figure 3D-F, compare with the control Figure 3A) away from the D/V boundary, which further suggests that the EcR pathway is normally required to restrict $w g$ expression to the D/V boundary. As previous reports had demonstrated that inhibition of the Wg pathway using TCF-dominant negative (DN) transgenes results in increased $\mathrm{S}$-phase across the $\mathrm{D} / \mathrm{V}$
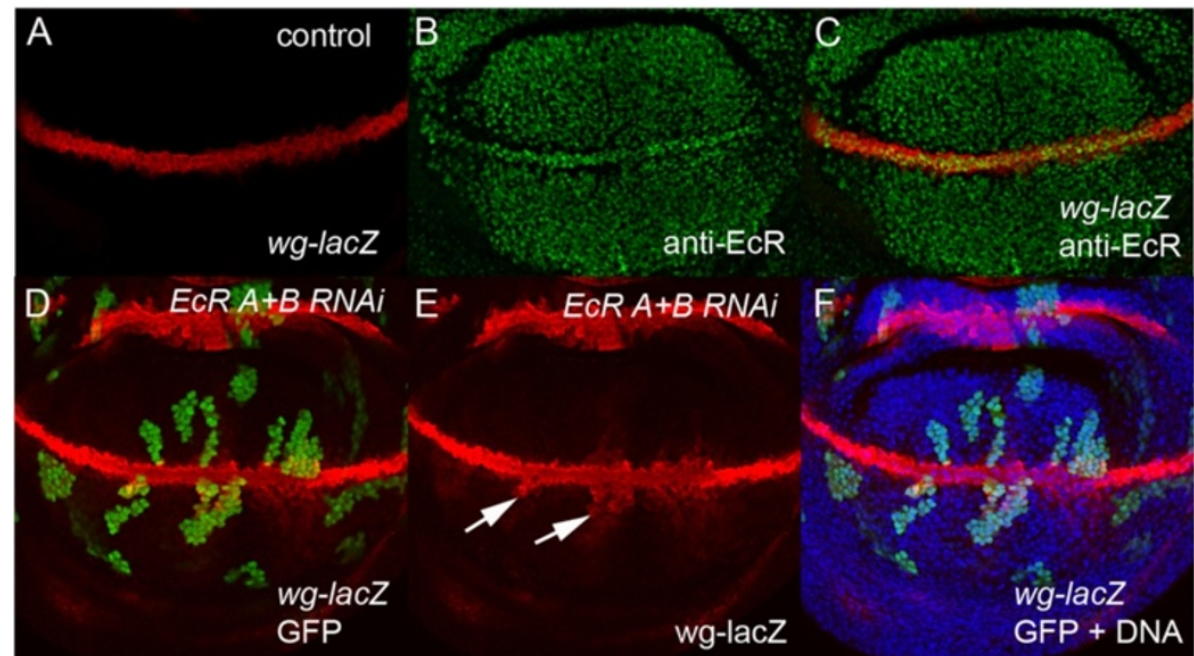

anti-ECR
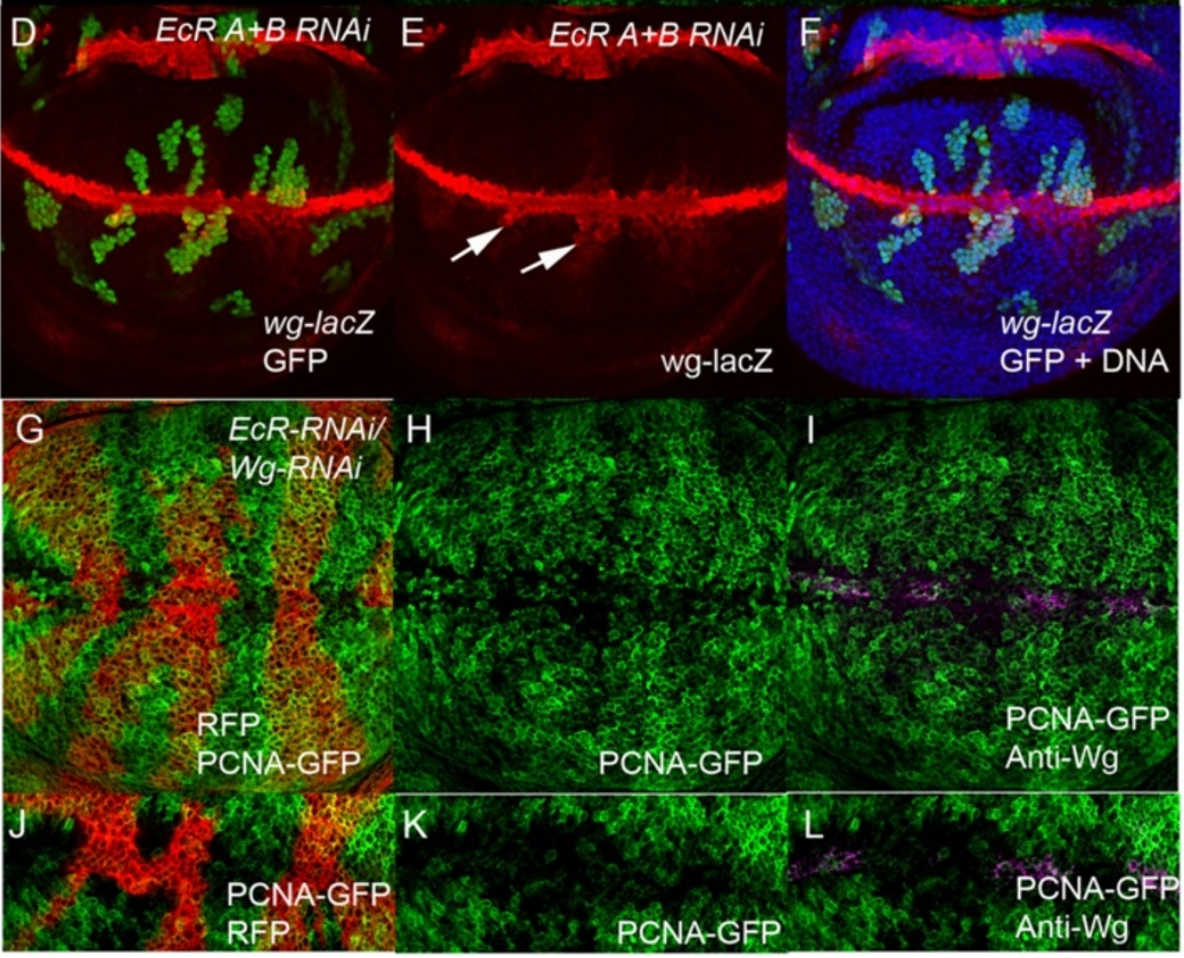

Anti-Wg

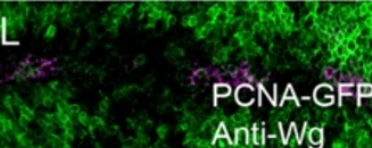

Figure 3 EcR pathway is required for Wg repression. (A-C) - EcR antibody staining (green) in the wg-lacZ background, detected with $\beta$-gal (red). (D-F) - wg-lacZ activity (red) in EcR RNAi clones marked with GFP. (G) - Control pattern of Wg antibody staining (red). (G-I) - shows wg RNAi and ECR RNAi double knockdown (marked with CD8-RFP) with PCNA-GFP patterning across the margin, while (J-L) shows a second example of wg RNAi and ECR RNAi co-knockdown focusing on the wing margin. 
boundary $[9,10]$, we tested whether the effect of EcR on E2F1 activity might be mediated by Wg. However, E2F1 patterning across the D/V boundary was not rescued by Wg knockdown in EcR RNAi clones (Figure 3G-K), compared with control $2 \mathrm{~A}$ and EcR knockdown alone in $2 \mathrm{E})$. To ascertain how loss of EcR might lead to disruption of E2F1 activity, we set out to determine whether EcR knockdown impacted other Wg cell cycle targets, including dMyc and Stg [9-11,13,14].

\section{EcR is not required for $\mathrm{dMyc}$ expression but is required for Stg repression across the margin}

$\mathrm{dMyc}$ is a key mediator of growth and S-phase progression in the wing imaginal disc [10] and previous work has shown that inhibition of Wg pathway activity is sufficient to ectopically activate $d m y c$ expression and S-phase throughout the wing margin $[10,11]$. We therefore tested whether loss of EcR might lead to ectopic E2F1 activity via effects on dMyc abundance using the dMyc antibody (from P. Bellosta and D. Grifoni [37]) on EcR RNAi clones (Additional file 1: Figure S1A-D). Although EcR knockdown across the margin results in ectopic E2F1 activity in the G2 band, levels of dMyc are not obviously altered in EcR RNAi clones spanning the G2 region, which suggests the expansion of the Wg domain in the EcR RNAi clones is unlikely to alter $S$ phase progression via $\mathrm{dMyc}$.

As Wg signaling also leads to down regulation of the essential mitotic regulator Cdc25 phosphatase String (Stg) across the G2 band of the margin at the level of transcription, we used a stg-lacZ enhancer trap to monitor stg promoter activity. Distribution of the $s t g-l a c Z$ enhancer trap and Wg protein shows stg promoter activity overlapping with Wg in the G1 cells of the margin, decreased in the G2 delayed cells, and abundant throughout the remainder of the pouch (Additional file 1: Figure S2A-D). Surprisingly, rather than leading to decreased stg promoter activity, as would be predicted given the expansion of the Wg domain in the EcR RNAi clones (Figure 3), EcR knockdown increases $s t g-l a c Z$ activity in clones spanning the margin (Additional file 1: Figure S2E-H). Together the data suggests that disruption to cell cycle patterning across the margin in the EcR RNAi clones is unlikely to be due to direct effects on dMyc, E2F or Stg.

\section{EcR is essential for CycB patterning across the wing margin}

The finding that dMyc is not altered and stg is ectopically expressed led us to investigate whether EcR might normally modulate cell cycle in the margin via the key G2-M cyclin, Cyclin B, which is also essential and ratelimiting for G2-M progression [38]. For this we first used a Cyclin B-GFP protein trap ( $C y c B-P T$, Carnegie collection CC01846, [39]) to monitor $C y c B$ expression in the wing. The $C y c B-P T$ reflects the pattern of $\mathrm{CycB}$ protein distribution in the wing (Figure 4A compare with anti-CycB in Additional file 1: Figure S3B) and the anti-EcR antibody and the $C y c B-P T$ overlap throughout the wing pouch (Figure 4A-D). The result of EcR knockdown is striking, with $E c R$ RNAi clones spanning the margin having dramatically decreased $C y c B-P T$ activity, particularly within the band of cells normally arrested in G2 (Figure 4E-H). To confirm that EcR RNAi also affects the distribution of $\mathrm{CycB}$ protein in a similar manner to the GFP-protein trap, we used the $\mathrm{CycB}$ antibody (From D. Glover [40]). In line with the $C y c B-P T$ data, EcR knockdown also results in decreased $\mathrm{CycB}$ protein across the margin (Additional file 1: Figure S3D-F).

The decreased $\mathrm{CycB}$ together with the elevated PCNAGFP further suggested that EcR RNAi clones spanning the G2 region of the margin were experiencing a G1 delay. To further investigate whether the G2 delay was disrupted in EcR loss of function cells at the margin, we co-stained for the DNA-replication inhibitor Geminin, which like $\mathrm{CycB}$ is usually abundant from the end of S-phase, peaks in G2 and is degraded at the anaphase-metaphase transition (Additional file 1: Figure S4G,I; [41]). Indeed, consistent with $E c R$ RNAi disrupting the G2 delay, we observe decreased Geminin in the presumptive G2 band, with G2 cells only observed at the position normally occupied by the G1 band (Figure 4I-L, marked by a white arrow). Together the cell cycle analysis for EcR RNAi clones suggests that EcR is normally required for expression of $\mathrm{CycB}$ (Figure 4), but for repression of Stg throughout this region of the margin (Additional file 1: Figure S2). To ensure that stg expression was not upregulated as a consequence of the decreased $\mathrm{CycB}$ in the EcR RNAi clones we generated $C y c B$ RNAi clones in the stg-lacZ background (Additional file 1: Figure S4A-C). As patterning of the $s t g$-lacZ reporter was not affected in $C y c B$ RNAi clones (compare with Additional file 1: Figure S2A-D), elevated stg promoter activity is unlikely to be an indirect consequence of $\mathrm{CycB}$ down regulation. Thus EcR is normally required to maintain cells in $\mathrm{G} 2$ via its ability to activate expression of $\mathrm{CycB}$, which is likely required for the final rounds of G2$\mathrm{M}$ progression across the wing margin. Moreover, the effect of the EcR pathway on $\mathrm{CycB}$ is specific to the margin and also results in non-autonomous affects on $\mathrm{CycB}$ in cells adjacent to clones spanning the margin (two sets of non-clonal tissue is outlined in white in Figure 4I-K), which suggests this might be mediated by expression of Wg across the D/V boundary.

\section{The cell cycle delay in EcR loss of function clones is dependent on $\mathrm{Wg}$ and $\mathrm{CycB}$}

As S-phase and mitosis are coupled in the wing [42], we speculated that the disruption to E2F1 activity in the $E c R$ RNAi clones might be an indirect consequence of the reduced abundance of $\mathrm{CycB}$, and therefore tested 


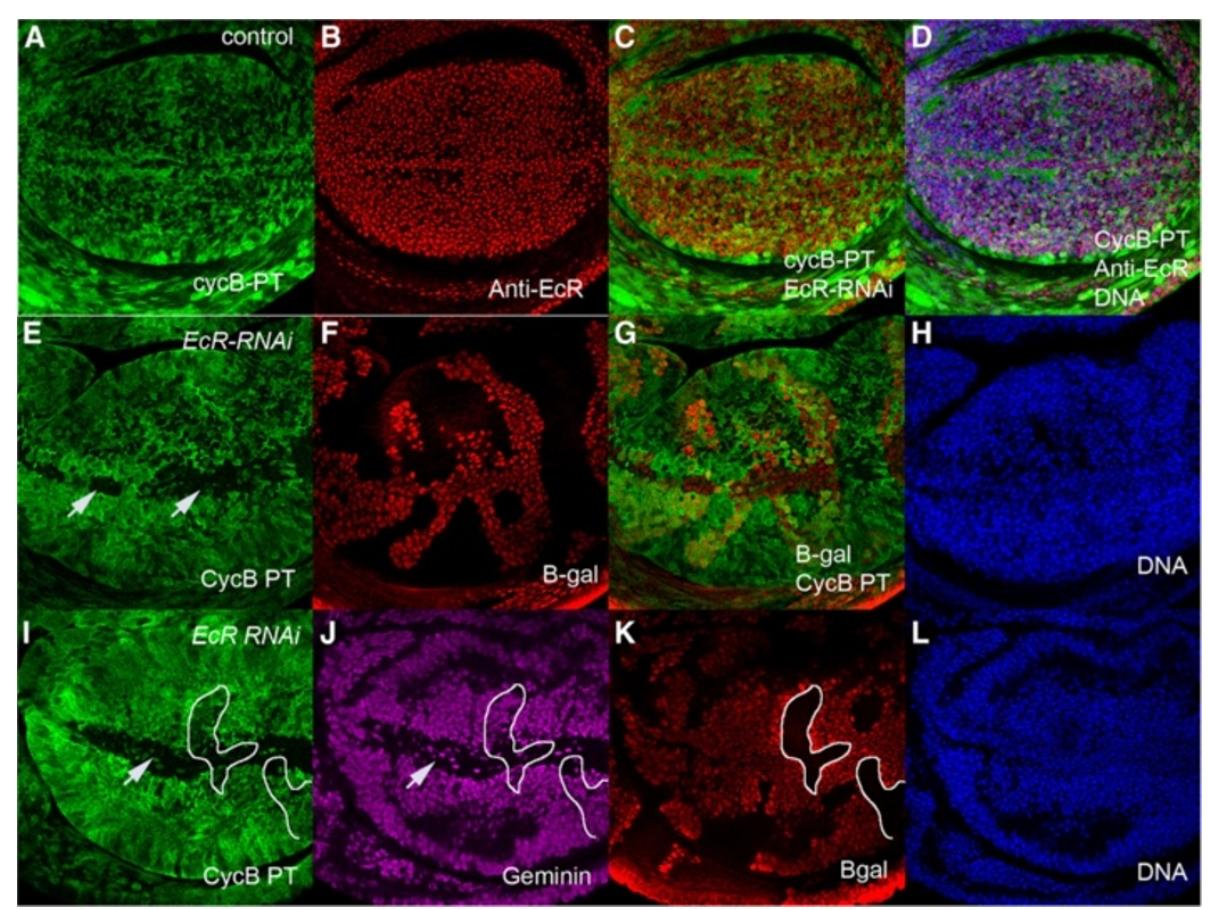

Figure 4 EcR is essential for CycB expression at the wing margin. (A-D) - Pattern of overlap between the Cyclin B-GFP protein trap (CycB-PT) in green (A) and anti-EcR antibody in red (B). The merge is shown in (C) and overlap with DNA in (D). (E-H) - EcR RNAi clones marked with $\beta$-gal (red) with CycB-PT (green). (E) - disruption to the Cyc B pattern across the margin marked with white arrows corresponds to the EcR RNAi clones shown with $\beta$-gal in $F$. The merge is shown in $\mathbf{G}$ and the presence of cells across the margin lacking CycB shown with DNA in $\mathbf{H}$. (I-L) -

Disruption to both CycB-PT activity in green (I) and the G2-marker Geminin in purple (J) for large ECR RNAi clones detected with $\beta$-gal in red (K). In (I-K) the position of two sets of non-EcR knockdown cells is show across the margin to highlight non-autonomous affects of EcR knockdown. The presence of cells across the margin shown with DNA in (L).

whether overexpression of $\mathrm{CycB}$ could restore E2F1 activity in the EcR RNAi clones (Figure 5). Overexpression of $C y c B$ in $E c R$ RNAi clones partially restored PCNAGFP patterning across the margin, with PCNA-GFP staining no longer decreased in the clones flanking the posterior margin and only occasional ectopic PCNAGFP cells in the G2 band of the anterior (Figure 5F, marked by an arrow). Together, this data is consistent with $\mathrm{CycB}$ being a key downstream target of EcR for normal patterning of cell cycle across the wing margin.

As EcR knockdown is associated with an expansion of the $\mathrm{Wg}$ domain and the disruption to $C y c B$ expression in $E c R$ RNAi clones only occurs around the D/V boundary where $\mathrm{Wg}$ is abundant, we tested whether we could restore $C y c B$ expression via co-knockdown of Wg. First, consistent with Wg normally being required for regulating $C y c B$ expression, the patterning of the $C y c B-P T$ was disrupted in $w g$ RNAi clones spanning the margin (Figure 5I-L). Within the $w g$ RNAi clones we observed unpatterned expression broadly across the margin, with ectopic $\mathrm{CycB}$ observed in the $\mathrm{G} 1$ band. In addition, we observed increased $\mathrm{CycB}$ adjacent to the large wg RNAi clone across the margin, consistent with Wg being required non-autonomously for repression of $\mathrm{CycB}$ (Figure 5J, white arrow). Strikingly, EcR knockdown only results in decreased $\mathrm{CycB}$ expression in the presence of $\mathrm{Wg}$, since $\mathrm{Wg}$ co-knockdown restores $C y c B-P T$ activity throughout EcR RNAi clones (Figure 5M-P, compare with Figure 4E,I). Therefore, in the absence of EcR, Wg is elevated in the G2 band and $\mathrm{CycB}$ expression is lost. However, in the absence of $\mathrm{Wg}$, down regulation of $\mathrm{CycB}$ no longer occurs in the EcR knockdown cells, which suggests $\mathrm{Wg}$ is required for EcR-dependent patterning of $\mathrm{CycB}$ at the margin. Thus we have identified a novel pathway for regulating cell cycle patterning across the margin, whereby EcR normally activates $\mathrm{CycB}$ in the $\mathrm{G} 2$ band by modulating the abundance of $\mathrm{Wg}$.

\section{Crol restores $w g$ repression in EcR loss of function cells}

The absence of EcR response elements in the $w g$ promoter suggests that $w g$ is unlikely to be a direct transcriptional target of EcR. We have previously shown that the ecdysone inducible-zinc finger transcription factor Crooked Legs (Crol) drives proliferation in Drosophila via effects on $w g$ expression [36]. Like EcR, Crol is normally expressed throughout the wing imaginal disc with reduced levels in the G2 cells of the margin [36]. Crol is responsive to the ecdysone pulse [35] and consistent 


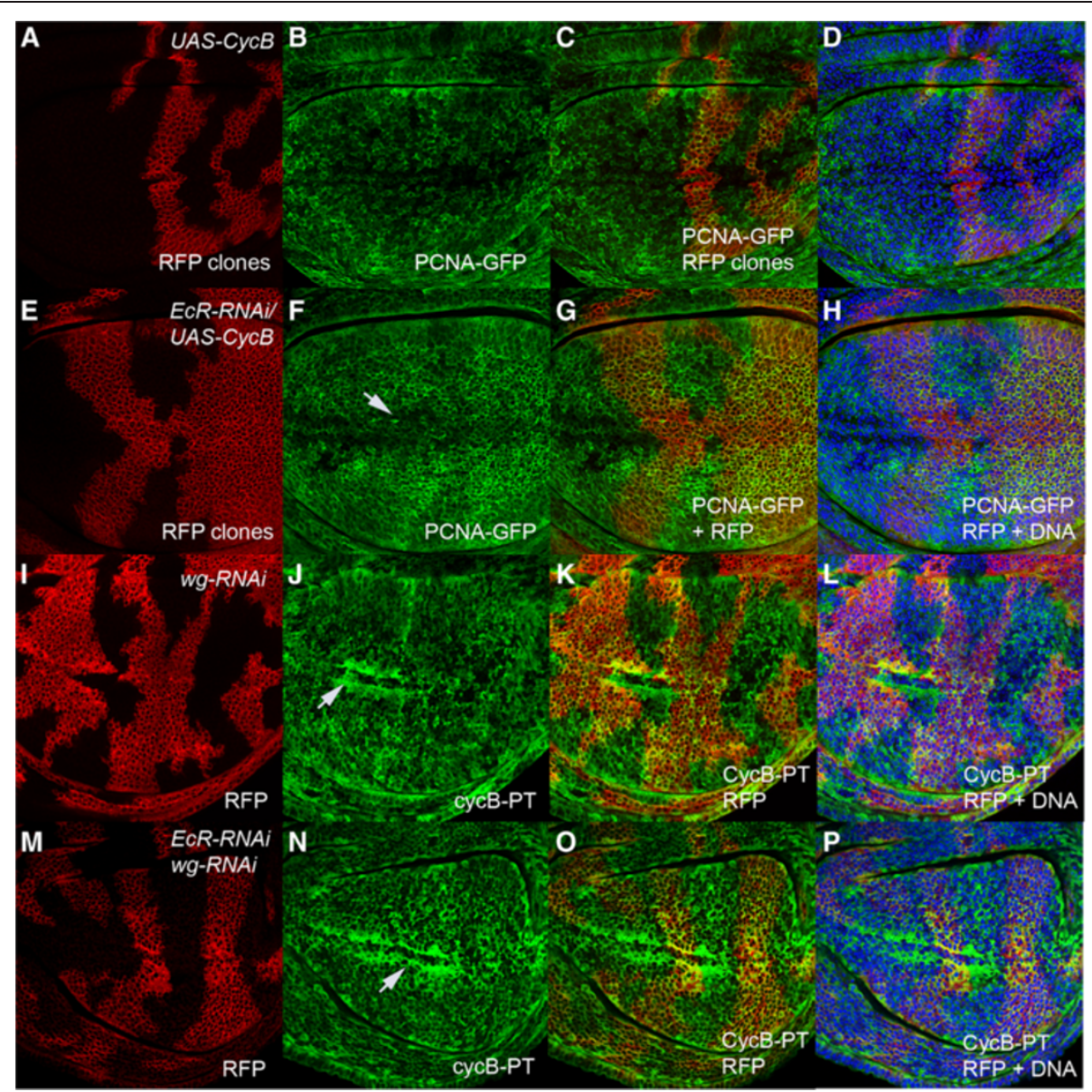

Figure 5 Overexpression of $\mathrm{CycB}$ or knockdown of $\mathbf{W g}$ partially restores cell cycle patterning in EcR RNAi clones. (A-D) - UAS-CycB clones (marked with CD8-RFP in A) do not disrupt PCNA-GFP patterning across the anterior margin (B). The merge is shown in (C) and overlap with DNA in D. (E-H) - Overexpression of CycB partially restores PCNA-GFP patterning (F, see arrow for remaining G1 cells) in EcR RNAi clones marked with CD8-RFP in (E). The merge is shown in (G) and overlap with DNA in (H). (I-L)- wg RNAi clones marked with CD8-RFP (I) disrupt CycB-PT patterning ( $\mathbf{J}$, see arrow). The merge is shown in (K) and overlap with DNA in (L). (M-P) - The double wg RNAi and ECR RNAi clones marked with CD8-RFP (M) result in restoration of CyCB-PT patterning (N). The merge is shown in $(\mathbf{O})$ and overlap with DNA in (P).

with the multiple $E c R$ binding sites in the crol promoter (data not shown), EcR is necessary for normal levels of crol expression (Figure 6A-B). In line with Crol being sufficient for $w g$ repression, overexpression of $\mathrm{crol}$ leads to disruption of the $w g$ expression domain and decreased $w g$-lacZ activity (Figure 6C-D). Although the wg-lacZ band was still disrupted when $\mathrm{Crol}$ is overexpressed in the EcR loss of function background, expansion of $w g$ lac $Z$ expression was no longer detected in clones away from the margin (Figure 6E-F). Thus overexpression of crol can partially restore $w g$-lacZ activity to the $\mathrm{D} / \mathrm{V}$ boundary in the EcR loss-of-function clones, which suggests EcR normally regulates $w g$ expression via Crol across the wing margin.

\section{Crol may mediate the effect of EcR on wg transcription} The data above suggests the zinc finger transcription factor Crol might provide a link between the ecdysone pulse and repression of $w g$ transcription across the wing margin. We have previously shown that Crol is unlikely to affect $w g$ transcription indirectly via effects on the Notch or Hh pathways [36] and, therefore, set out to test whether Crol might directly inhibit $w g$ transcription in larval imaginal tissue by conducting ChIP with overlapping primer sets spanning the $<5 \mathrm{~kb} w g$ promoter (Figure 7 ). As reporter constructs corresponding to the region covered by the first 3 primer sets $(-4600$ to -2614$)$ had been previously shown to be bound by $\mathrm{Ci}$ in Drosophila S2 cells in vitro [43-45], we carried out ChIP with these primer sets using the $\mathrm{Ci}$ antibody as a positive control. We detected enrichment for $\mathrm{Ci}$ using primer set Wg2, but not on primer set Wg1 or Wg3, which narrows down the binding to between -3750 and -3462 of the $w g$ promoter (Figure 7A, C). ChIP carried out for Crol, revealed enrichment for overlapping primer sets Wg2 and Wg3 (Figure 7A, C), suggesting Crol binds the $w g$ promoter between -3750 


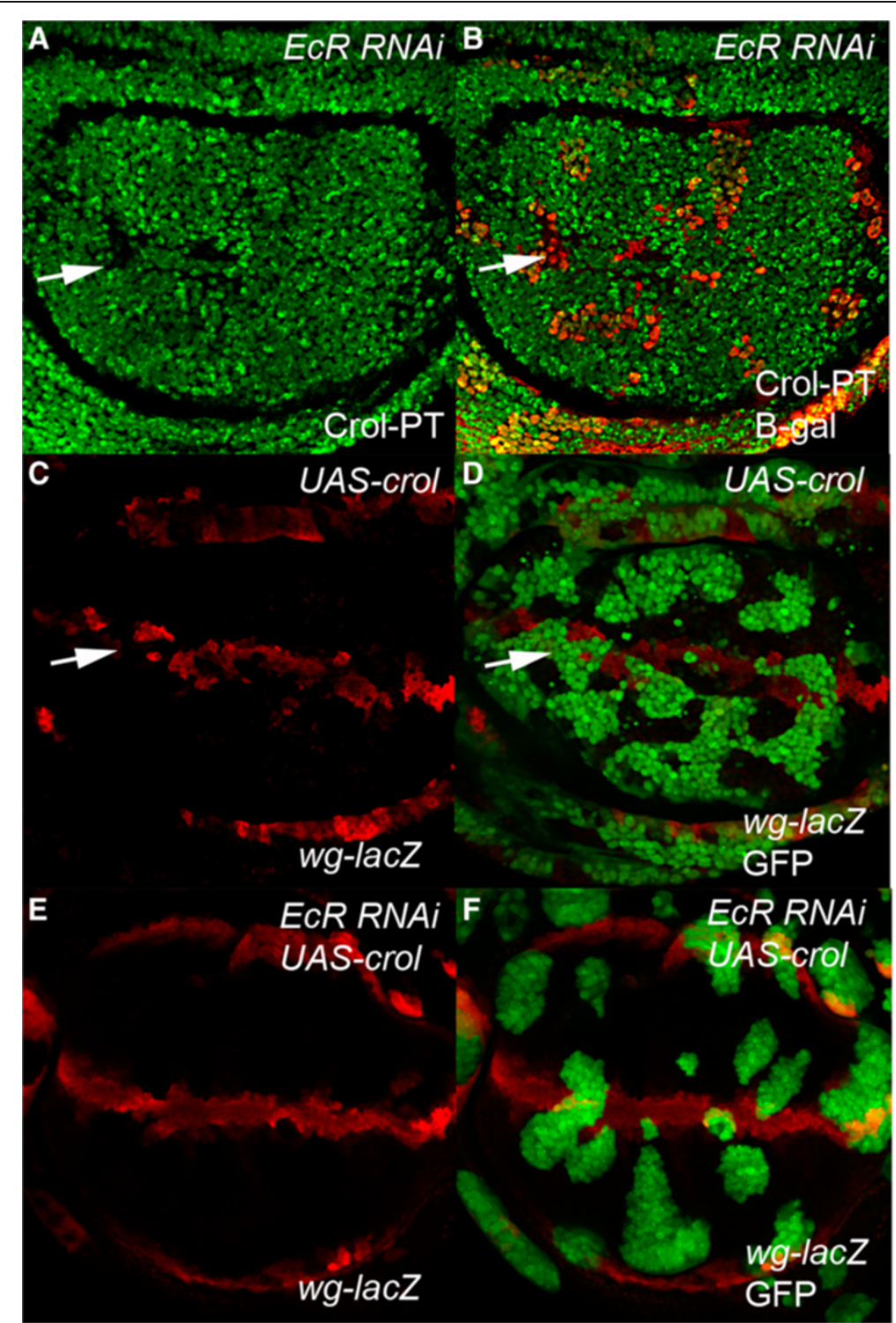

Figure 6 Crol restores repression of $w g$ expression in EcR knockdown. (A-B) - Crol-GFP protein trap activity (A) is reduced in ECR RNA clones marked with $\beta$-gal in red for the merge (B). (C-D) - wg-lacZ activity marked with $\beta$-gal in red (C) is reduced in UAS-crol clones marked with GFP in the merge (D). (E-F) - UAS-crol restores repression of wg-lacZ activity in the ECR RNAi clones.

and -2614 . Analysis of the 1160 bp sequence contained within this portion of the $w g$ promoter using the Zinc Finger Tools (ZF Tools) website (http://www.zincfingertools. org) to identify contiguous sites with a minimum target size of 24 bp (for 8 finger binding) [46] revealed 2 strong consensus zinc finger binding sites within this region of the $w g$ promoter (-3682, -3656 boxed in Figure 7B).

To determine whether $\mathrm{Crol}$ or $\mathrm{Ci}$ might normally bind these consensus zinc-finger binding sites we designed overlapping primer sets for ChIP-qPCR for this $1160 \mathrm{bp}$ region i.e. between -3750 and -2614 (Wg2.1-Wg2.4 shown in Figure 7B). Significant enrichment was observed for both $\mathrm{Crol}$ and $\mathrm{Ci}$ across the 2 most 5' amplicons (Wg2.1 and Wg2.2 primers) in the region containing 2 zinc finger consensus sites, compared with the more 3' amplicons (Figure 7D,E). Thus the $w g$-repressor $\mathrm{Crol}$ and the $w g$-activator $\mathrm{Ci}$ are enriched within overlapping regions of the $w g$-promoter and it will be of future interest to determine whether the fine-tuning of $w g$ expression involves interaction between $\mathrm{Ci}$ and $\mathrm{Crol}$. Together this data suggests that EcR normally maintains cell cycle across the wing margin by activating expression of the Crol transcription factor, which in turn binds the $w g$ promoter to repress transcription (Figure 7F). Thus we have identified a critical function for the ecdysone pathway in refining expression of the Wg 


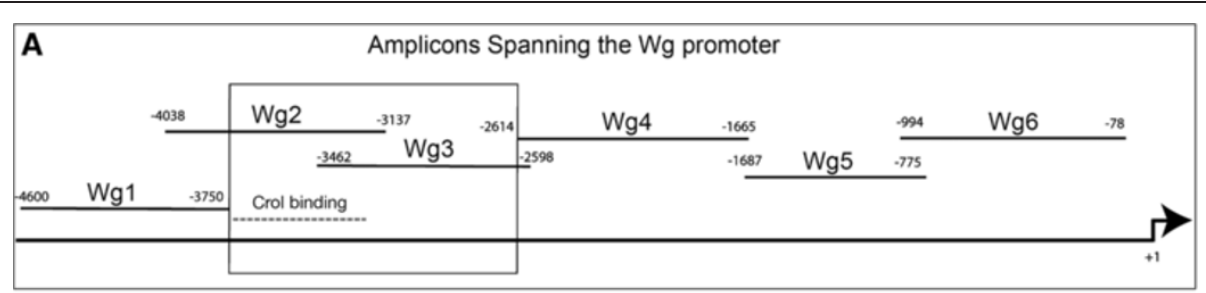

B Wg promoter -3750 to -3290
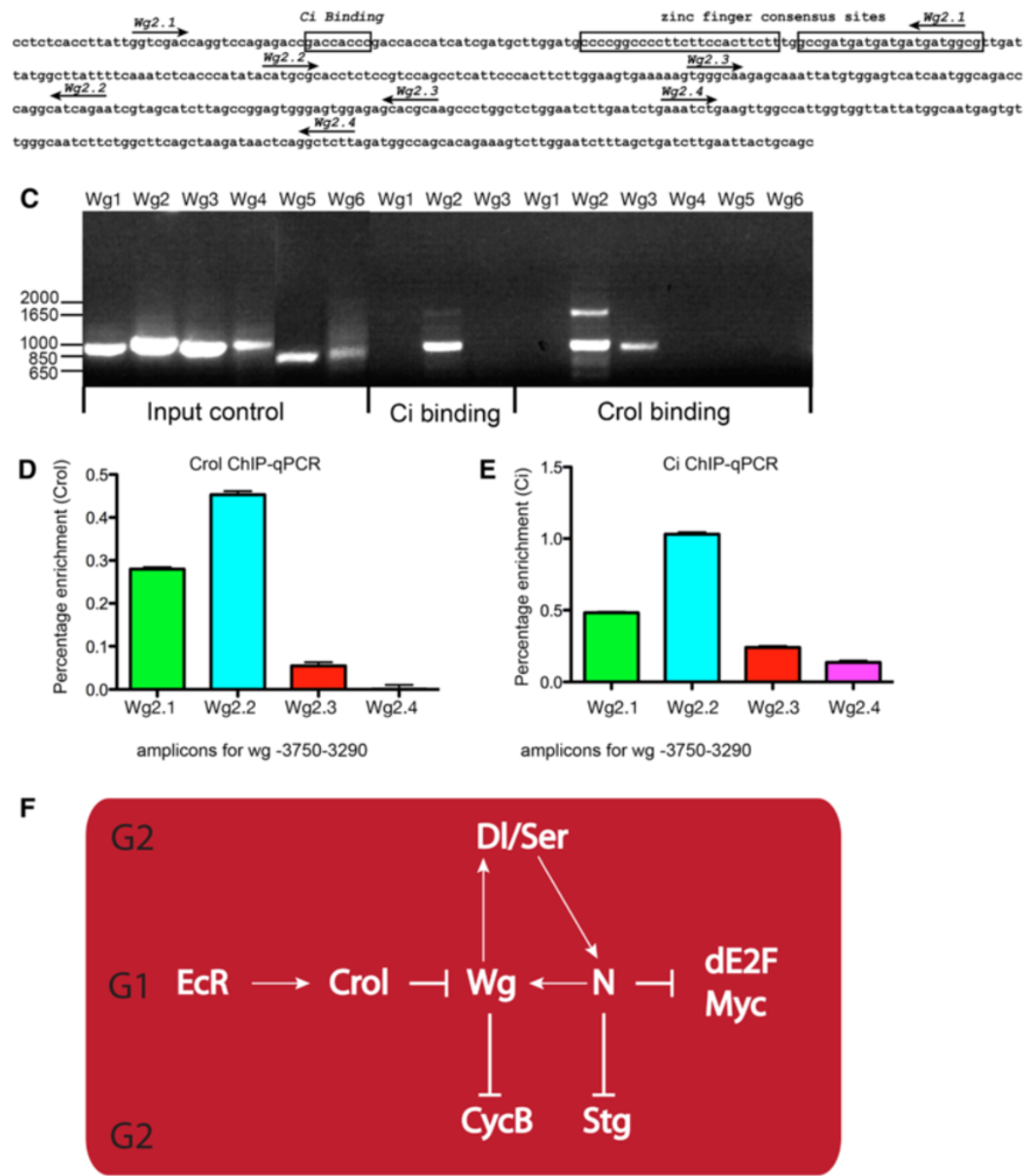

Figure $7 \mathrm{Crol}$ is enriched across consensus zinc finger binding sites in the $\boldsymbol{w g}$ promoter. (A) - 6 overlapping primer sets spanning $5 \mathrm{~kb}$ of the wg promoter, wg transcription start site +1. (B) - region of wg promoter containing overlapping amplicons for wg (Wg primers 1-6) used for ChIP with anti-Crol or $\mathrm{Ci}$, the boxed region highlights amplicons positive for $\mathrm{Crol}$ enrichment (see gel in $\mathbf{C}$ ) and the dashed grey line shows the region of the wg promoter containing the zinc finger and Ci consensus sites shown in 7B. (B) - Position of primers for ChIP-qPCR (Wg2.1-Wg2.4) are marked with arrows. The in vitro defined Ci binding site and consensus zinc finger binding sites are boxed and labelled. (C) - DNA gel electrophoresis of ChIP-PCR across the Wg promoter using Wg primers (as marked in 7) for Input DNA; Ci ChIP or Crol ChIP as marked. (D-E) Fine mapping of Crol and Ci enrichment using ChIP-qPCR for small amplicons - Wg2.1-2.4 shown in (B). (D) - ChIP for Crol - percentage enrichment and error for percentage enrichment for Crol are as follows; Wg $2.1(0.28 \pm 0.0045) ; \mathrm{Wg} 2.2(0.453 \pm 0.0083) ; \mathrm{Wg} 2.3(0.055 \pm 0.0082)$ and Wg 2.4 (0.00116 \pm 0.0095). (E) - ChIP for $\mathrm{Ci}$ - percentage enrichment and Error for percentage enrichment for Ci are as follows; Wg 2.1 (0.482 \pm 0.0066$)$; Wg $2.2(1.031 \pm 0.0141)$; Wg $2.3(0.24 \pm 0.0103)$ and Wg $2.4(0.137 \pm 0.019)$. (F) - Model for regulation of cell cycle patterning across the anterior wing margin by EcR. 
morphogen and, as a consequence, ensuring proper timing of cell cycle exit at the margin via expression of the key mitotic regulator $\mathrm{CycB}$.

\section{Discussion}

We have identified the essential mitotic cyclin, $\mathrm{CycB}$ as the key target of the ecdysone/EcR system that normally ensures cells remain competent to complete their final divisions during the early pupal stage by activating expression of the Crol transcription factor (Figure 7F). Early studies demonstrated that $\mathrm{Crol}$ is activated in late larval imaginal discs by the steroid hormone ecdysone [35]. More recently Crol was identified in a genetic screen for factors capable of disrupting the establishment and/or maintenance of heterochromatic silencing in either cycling and differentiated cells [47], consistent with a transcriptional role for $\mathrm{Crol}$ in maintaining active chromatin states of many genes specific to cycling cells. Although this screen identified many chromatin and RNA processing factors capable of de-repressing silencing, most factors behaved as de-repressors in both cycling and differentiated cells. Interestingly, only two factors, Crol and the bantam micro RNA, were found to de-repress silencing in cycling, but not differentiated cells, both of which have now been implicated in regulating cell cycle across the wing margin (this work, $[11,36,48]$ ).

The rise of ecdysone levels at the end of the third larval instar [1] coincides with reduced rates of cell division across the wing disc $[5,6]$ and increased expression of proneural genes in clusters of cells, which then form the sensory organ precursor (SOP) lineage required for bristles development along the wing margin $[7,8]$. At the beginning of the pupal period, the bristle precursors complete their final cell divisions and by 24 hours APF all epithelial cells have arrested in G1 [5,6]. Our data suggests that loss of EcR across the margin results in a premature arrest of these cells in G1. EcR is therefore required for coordinating the delay in cell cycle progression with differentiation of the sensory neurons during wing metamorphosis. Indeed previous studies have shown that EcR is required for repressing the differentiation of the SOPs in the wing margin, as RNAi results in ectopic senseless expression [34]. The finding that sensory neurons in the margin differentiate precociously is consistent with our findings that suggest EcR coordinates the onset of sensory neuron development by maintaining cell cycle gene expression across the margin. Furthermore, in the studies by Schubiger et al. (2005), loss-of-function EcR only resulted in precocious differentiation of SOPs not ectopic differentiation, which suggests that this is mediated by a second factor normally expressed across the margin, and our work suggest that this is likely to be Wg. Consistent with this idea, ectopic activation of the $\mathrm{Wg}$ pathway also results in ectopic SOPs [21]. The data here demonstrates Wg is a critical target in mediating the effect of the EcR signaling pathway on cell cycle patterning in the wing imaginal disc. Inhibition of the EcR pathway results in ectopic $w g$ transcription and expansion of the band of $\mathrm{Wg}$ protein comprising the dorsal-ventral boundary of the wing imaginal disc. Thus EcR is normally required for constraining the expression of $\mathrm{Wg}$ to the $\mathrm{D} / \mathrm{V}$ boundary of the wing imaginal disc in order to pattern the final rounds of cell division required for differentiation and development of the wing margin.

Previous work has demonstrated that the G2 cell cycle delay across the wing imaginal disc is mediated by down regulation of Stg in response to activity of the $\mathrm{Wg}$ and Notch pathways $[9,11]$. At the D/V boundary, Notch can activate $w g$ expression $[15,17]$ and $\mathrm{Wg}$ signaling activates $a c / s c$ to lead to down regulation of stg and delay of the cells flanking the boundary in G2 [9]. Thus we originally predicted that $E c R$ RNAi clones, which show an expansion of Wg away from the margin, might display down regulation of Stg. Conversely, we observed ectopic Stg in the G2 band of the margin, which raises the question of how expansion of the Wg domain in the EcR knockdown result in ectopic $s t g$ ? This could be due to the ability of Wg to repress $\mathrm{N}$ away from the $\mathrm{D} / \mathrm{V}$ boundary $[11,48]$, whereby the expansion of $\mathrm{Wg}$ in the EcR knockdown might result in ectopic Notch activity, which would lead to repression of $a c / s c$ expression and up regulation of stg in the nonboundary G2 band [11]. In the EcR RNAi clones, Notch would still be abundant at the D/V boundary to autonomously delay cells in G1, via down regulation of dMyc and Bantam, however, in the absence of EcR these cells would be unable to progress through G2-M due to the decreased expression of $\mathrm{CycB}$ (Figures 2 and 4). Furthermore previous work has shown that stg overexpression only triggers cell-cycle progression in embryonic and imaginal cells previously arrested in G2 [5,49,50], but not in G1-arrested cells [51]. Thus the cells spanning the margin in the EcR knockdown are predominantly delayed in G1. The interplay between EcR/Wg and N/Wg is therefore likely to be essential for orchestrating the cell cycle exit across the presumptive margin, which is required for sensory neuron differentiation and development of the wing margin.

Ecdysone-dependent control mechanisms have been reported for restricting growth to the juvenile period, where ecdysone controls growth rate via dMyc in the fat body [27]. In contrast to the role of EcR as a CycB activator in the wing margin, EcR signaling normally represses $\mathrm{dMyc}$ and its downstream targets in the fat body. Furthermore, the ability of circulating ecdysone to control dMyc expression during the pupal stage was found to be specific to the fat body. For example, dmyc mRNA levels were elevated in fat body after reducing the level of circulating ecdysone, via inhibition of PI3K 
pathway in the prothoracic gland, but at this stage global changes to $d m y c$ levels were not detected in wing imaginal discs [27]. Consistent with this, we did not observe changes in dMyc levels in EcR RNAi clones throughout the third instar wing (Additional file 1: Figure S1B-C). Thus the effect of ecdysone on this key growth regulator appears to be tissue specific; resulting in down regulation of $d m y c$ expression in the fat body, but not in the wing disc either during the larval or pupal stage.

The ecdysone pulse at the larval-pupal transition is required for the stg transcription triggering histoblast proliferation at the onset of abdomen metamorphosis [26]. In contrast to the wing epithelium, during larval stages histoblasts grow in a G2 arrested state prior to entering a proliferative stage during pupal metamorphosis [25,52]. During larval stages, the arrested histoblasts accumulate cellular mass and the transition to a proliferative state is initiated by ecdysone-dependent stg transcription [26]. The latter can occur because the larval histoblasts have preaccumulated stores of the G1 cyclin, Cyclin E, which is sufficient to trigger S-phase after mitosis. In the histoblast, overexpression of Stg, but not Cyclin A, Cyclin B, or Cdk1, can trigger their premature hyper proliferation in larval stages [26]. Although ecdysone is necessary to trigger histoblast proliferation [23] up regulation of stg transcription in larval stages bypasses the requirement for ecdysone pathway activity. Thus ecdysone is important for coupling growth and proliferation in abdominal histoblasts [26].

It will be of interest to determine whether the changes reported in stg or dmyc are due to direct transcriptional effects of EcR or are mediated by changes to developmental signaling. Our findings in the imaginal tissues suggest that EcR might regulate cell cycle genes in larval histoblasts and fat body indirectly, by modulating upstream developmental signaling pathways. As noted by Delanoue (2010), the lack of consensus binding sites for EcR/Usp (EcREs) in the $d m y c$ promoter region suggests that $d m y c$ is not a direct target of EcR-mediated gene repression in the fat body, but rather that EcR signaling indirectly controls $d m y c$ transcription. Although the fatspecific target of EcR leading to altered dmyc expression is unknown, here we have shown that in the wing imaginal disc EcR can activate the Wg-repressor Crol, which is required for repression of the $\mathrm{Wg}$ morphogen.

\section{Conclusions}

Despite evolutionary divergence, functional studies suggest that the estrogen steroid hormone pathway is functionally similar to the ecdysone pathway [53-55]. Aberrant estrogen signaling is associated with a variety of hormonedependent diseases, including cancer [56]. Moreover, in human colon cancer, estrogen receptor (ER) signaling is documented as an inhibitor of the Wnt pathway [57], which has long been implicated in initiation and progression of colorectal cancer [58], however, the mechanism by which estrogen modulates Wnt signaling is currently unknown. Our discovery that EcR represses the Wg pathway via an intermediate zinc-finger transcription factor may, therefore, have broader implications for studies of Wnt pathway regulation by estrogen/ ER in colorectal cancer.

\section{Methods \\ Drosophila strains}

The following fly stocks were obtained from the Bloomington Stock Centre; UAS-CycB; wg-lacZ (P\{en1\}wg en11; [59]); UAS-EcR RNAi [34]. wg-RNAi (v13351; v13352) was obtained from the Vienna Drosophila RNAi Centre, http://www.vdrc.at/ [60]. The $C y c B$ (CC01846) and Crol (CB 03039) GFP protein trap lines were from The Carnegie Fly Trap collection [39]. All other lines were as follows; Act < CD2 < GAL4 UAS-GFP (L. Johnston), PCNA-GFP (R. Duronio) and stg-lacZ (P(2) Stg 01235) (Sveged, S11525), UAS-crol transgenic lines (Mitchell et al., 2008).

\section{Immunohistochemistry and microscopy}

For all flip-out clones larvae were heatshocked for 20 minutes at $37^{\circ} \mathrm{C} 48$ hours after egg deposition. Larvae were raised at $25^{\circ} \mathrm{C}$ for 72 hours to allow development to the third larval instars prior to dissection. All other larvae were dissected 120 hours after egg deposition. Antibody staining was carried out as described previously [36,61]. Antibodies used were: Cyclin B (D. Glover; [40]), anti-Geminin [41], anti- $\beta$ gal (Sigma), anti-GFP (to detect PCNA-GFP only, Invitrogen), Anti-Wingless 4D4 (DSHB), EcR common (DSHB). Image preparation and analysis are conducted in Adobe Photoshop CS2 Version 9.0 ㄷ, ImageJ.

\section{ChIP across wg promoter}

ChIP was carried out using the ChIP Assay Kit (Upstate Biotech) as described previously [61]. For each ChIP sample (for PCR or qPCR), 200 larval heads were fixed in 4\% paraformaldehyde in PBS for 40 minutes. ChIP followed by PCR was first carried across the entire $w g$ promoter after sonication for genomic fragments between 400bp$1 \mathrm{~kb}$ (not shown). IP was carried out using either $\mathrm{Ci}$ or the Crol antibody, which we have shown to specifically detect Crol protein [36]. The primers used for PCR to test binding of $\mathrm{Crol}$ and $\mathrm{Ci}$ across the 4625bp span of the $w g$ promoter were; Primer set 1 ( 827 bp product -4600 to -3750 ) $5^{\prime}$ agcgtggacgatgataatgc 3 ' and 5' tcgaccaataaggtgagagg $3^{\prime}$; Primer set 2 (901 bp product -4038 to -3137 ) 5' aagtgcgtgaaccatgtcg $3^{\prime}$ and $5^{\prime}$ tggcagatcaacaccattagg $3^{\prime}$; Primer set 3 (864bp product -3462 to -2598 ) $5^{\prime}$ ggccattggtggttattatgg $3^{\prime}$ and $5^{\prime}$ tgtcctgtcagcagaattgg $3^{\prime}$; Primer set 4 (949bp product -2614 to -1665 ) 5' 
ttctgctgacaggacacagc $3^{\prime}$ and $5^{\prime}$ atggcagatagcgagagtgg $3^{\prime}$; Primer set 5 (726bp product -1687 to -775$) 5^{\prime}$ ccaccactctcgctatctgc $3^{\prime}$ to $5^{\prime}$ ttcctcatccattgcttgg $3^{\prime}$; Primer set 6 (826bp product -994 to -78 ) $5^{\prime}$ gccaagcaatggatgagg $3^{\prime}$ to $5^{\prime}$ ggtcttcgtcttcggatcg 3 '.

For ChIP-qPCR 200 heads were prepared as above, but sonication was performed to generate $100 \mathrm{bp}$ genomic fragments (not shown). Quantitative real-time PCR (q-PCR) was carried out in triplicate using the SYBR Green PCR Master Mix (Applied Bio systems) and the 7900HT Fast Real-Time PCR System (Applied Bioscience). The primers for qPCR were; qPCR Primer set wg2.1 - 5' ggtcgaccaggtccagagaccg $3^{\prime}$ and 5'cgccat catcatcatcatcggccaaa 3'; qPCR Primer set wg2.2 - 5' catgcgcacctctccgtccag $3^{\prime}$ to $5^{\prime}$ tctgatgcctgggtctgcca $3^{\prime}$; qPCR Primer set wg2.3 - 5' tgggcaagagcaaattatgtggagtca $3^{\prime}$ to $5^{\prime}$ ccagagccagggcttgcgtg $3{ }^{\prime}$; qPCR Primer set wg2.4 - 5' aaatctgaagttggccattggtggt $3^{\prime}$ to $5^{\prime}$ tctgtgc tggccatctaagagcct $3^{\prime}$ (the position of each primer in the refined region of Crol binding is shown in Figure 7). The data analysis was conducted with Sequence Detection Systems v2.3 (Applied Bio systems). Enrichment was determined by normalising signal to input using the "Percent Input Method" [62], which includes normalization for both background levels and input chromatin. The input sample was purified, non-immuno precipitated, sheared chromatin; target was immuno precipitated sheared chromatin; and background was from an immunoprecipitation with non-specific IgG antibody. To normalise to background, the signal obtained for the nonspecific IgG for each primer set was subtracted from the ChIP signal from either the $\mathrm{Ci}$ or Crol antibody.

\section{Additional file}

Additional file 1: Figure S1. dMyc protein is not decreased in EcR knockdown. A - dMyc antibody staining on control. B-D - ECR RNAi clones with anti-dMyc staining. Figure S2. EcR is required to represses stg in the margin. A-D - Wg staining in stg-lacZ enhancer trap background. E-H - ECR RNAi clones in the stg-lacZ background. Figure S3. EcR overexpression disrupts $C y c B$ patterning across the margin. A-C - costaining of control wing dics with Wg and CycB. D-F - ECR RNAi clones stained with $\mathrm{CycB}$ antibody. G-H - co-staining for Geminin and Cyclin B across the wing imaginal disc. Figure S4. CycB knockdown does not affect stg-lacZ activity across the wing margin. A-C - CycB RNAi clones in the stg-lacZ background.

\section{Competing interests}

The authors have no financial or non-financial competing interests.

\section{Authors' contributions}

$\mathrm{NM}, J \mathrm{~L}, \mathrm{OZ}, \mathrm{NC}, \mathrm{AL}$ and $\mathrm{LQ}$ conducted experiments. LQ wrote the manuscript. All authors read and approved the final manuscript.

\section{Acknowledgments}

This work was supported by the Australian National Health and Medical Research Council (NHMRC) and Cancer Council Victoria (CCV). LQ, NM and $\mathrm{L}$ are supported by NHMRC project grants. NC is supported by an Australian Postgraduate Award (APA). AL is supported by a Victorian Government
International Scholarship. We thank the following researchers for reagents Laura Johnston (UAS-myc and Act < CD2 < GAL4 UAS-GFP), Bruce Edgar (UAS-E2F1/UAS-DP), Robert Duronio (PCNA-GFP), Daniella Grifoni and Paola Bellosta (dMyc antibody), David Glover (Cyclin B antibody). We thank the Vienna Drosophila Resource Centre (VDRC), National Institute of Genetics, Japan (NIG), the Bloomington Stock Center, Sveged and The Carnegie Fly Trap collection for Drosophila strains and the Developmental Hybridoma Bank (DSHB) for antibodies.

Received: 25 March 2013 Accepted: 10 July 2013

Published: 13 July 2013

\section{References}

1. Thummel CS: Molecular mechanisms of developmental timing in C. Elegans and Drosophila. Dev Cell 2001, 1:453-465.

2. Thummel CS: Files on steroids-Drosophila metamorphosis and the mechanisms of steroid hormone action. Trends Genet 1996, 12:306-310.

3. Zitnan D, Kim YJ, Zitnanova I, Roller L, Adams ME: Complex steroid-peptidereceptor cascade controls insect ecdysis. Gen Comp Endocrinol 2007, 153:88-96.

4. McBrayer Z, Ono H, Shimell M, Parvy JP, Beckstead RB, Warren JT, Thummel CS, Dauphin-Villemant C, Gilbert LI, O'Connor MB: Prothoracicotropic hormone regulates developmental timing and body size in Drosophila. Dev Cell 2007, 13:857-871.

5. Milan M, Campuzano S, Garcia-Bellido A: Cell cycling and patterned cell proliferation in the Drosophila wing during metamorphosis. Proc Natl Acad Sci USA 1996, 93:11687-11692.

6. Buttitta LA, Katzaroff AJ, Perez CL, Dela Cruz A, Edgar BA: A doubleassurance mechanism controls cell cycle exit upon terminal differentiation in Drosophila. Dev Cell 2007, 12:631-643.

7. Skeath JB, Carroll SB: Regulation of achaete-scute gene expression and sensory organ pattern formation in the Drosophila wing. Genes Dev 1991, 5:984-995.

8. Cubas P, de Celis JF, Campuzano S, Modolell J: Proneural clusters of achaete-scute expression and the generation of sensory organs in the Drosophila imaginal wing disc. Genes Dev 1991, 5:996-1008.

9. Johnston LA, Edgar BA: Wingless and notch regulate cell-cycle arrest in the developing Drosophila wing. Nature 1998, 394:82-84.

10. Johnston LA, Prober DA, Edgar BA, Eisenman RN, Gallant P: Drosophila myc regulates cellular growth during development. Cell 1999, 98:779-790.

11. Herranz H, Pérez L, Martín FA, Milán M: A wingless and notch doublerepression mechanism regulates G1-S transition in the Drosophila wing. EMBO J 2008, 27:1633-1645.

12. Baker NE: Patterning signals and proliferation in Drosophila imaginal discs. Curr Opin Genet Dev 2007, 17(7):287-293.

13. Duman-Scheel M, Johnston LA, Du W: Repression of dMyc expression by wingless promotes Rbf-induced G1 arrest in the presumptive Drosophila wing margin. Proc Natl Acad Sci USA 2004, 101:3857-3862.

14. Johnston LA, Sanders AL: Wingless promotes cell survival but constrains growth during Drosophila wing development. Nat Cell Bio/ 2003, 5:827-833.

15. Diaz-Benjumea FJ, Cohen SM: Serrate signals through notch to establish a wingless-dependent organizer at the dorsal/ventral compartment boundary of the Drosophila wing. Development 1995, 121:4215-4225.

16. Neumann CJ, Cohen SM: Distinct mitogenic and cell fate specification functions of wingless in different regions of the wing. Development 1996, 122:1781-1789.

17. de Celis JF, Garcia-Bellido A, Bray SJ: Activation and function of notch at the dorsal-ventral boundary of the wing imaginal disc. Development 1996, 122:359-369.

18. Rulifson EJ, Micchelli CA, Axelrod JD, Perrimon N, Blair SS: Wingless refines its own expression domain on the Drosophila wing margin. Nature 1996, 384:72-74.

19. Axelrod JD, Matsuno K, Artavanis-Tsakonas S, Perrimon N: Interaction between wingless and notch signaling pathways mediated by dishevelled. Science 1996, 271:1826-1832.

20. de Celis JF, Bray S: Feed-back mechanisms affecting notch activation at the dorsoventral boundary in the Drosophila wing. Development 1997, 124:3241-3251.

21. Baena-López LA, Franch-Marro X, Vincent J-P: Wingless promotes proliferative growth in a gradient-independent manner. Sci Signal 2009, 2:ra60. 
22. Li TR, White KP: Tissue-specific gene expression and ecdysone-regulated genomic networks in Drosophila. Dev Cell 2003, 5:59-72.

23. Ninov N, Chiarelli DA, Martin-Blanco E: Extrinsic and intrinsic mechanisms directing epithelial cell sheet replacement during Drosophila metamorphosis. Development 2007, 134:367-379.

24. Lawrence PA, Casal J, Struhl G: The hedgehog morphogen and gradients of cell affinity in the abdomen of Drosophila. Development 1999, 126:2441-2449.

25. Lawrence PA, Casal J, Struhl G: Hedgehog and engrailed: pattern formation and polarity in the Drosophila abdomen. Development 1999 126:2431-2439.

26. Ninov N, Manjon C, Martin-Blanco E: Dynamic control of cell cycle and growth coupling by ecdysone, EGFR, and PI3K signaling in Drosophila histoblasts. PLoS Biol 2009, 7:e1000079.

27. Delanoue R, Slaidina M, Leopold P: The steroid hormone ecdysone controls systemic growth by repressing dMyc function in Drosophila fat cells. Dev Cell 2010, 18:1012-1021.

28. Lin Jl, Mitchell NC, Kalcina M, Tchoubrieva E, Stewart MJ, Marygold SJ, Walker CD, Thomas G, Leevers SJ, Pearson RB, Quinn LM, Hannan RD: Drosophila Ribosomal protein mutants control tissue growth Nonautonomously via effects on the prothoracic gland and ecdysone. PLoS Genet 2011, 7:e1002408.

29. Caldwell PE, Walkiewicz M, Stern M: Ras activity in the Drosophila prothoracic gland regulates body size and developmental rate via ecdysone release. Curr Biol 2005, 15:1785-1795.

30. Orme MH, Leevers SJ: Flies on steroids: the interplay between ecdysone and insulin signaling. Cell Metab 2005, 2:277-278.

31. Thacker SA, Bonnette PC, Duronio RJ: The contribution of E2F-regulated transcription to Drosophila PCNA gene function. Curr Biol 2003, 13:53-58.

32. Talbot WS, Swyryd EA, Hogness DS: Drosophila tissues with different metamorphic responses to ecdysone express different ecdysone receptor isoforms. Cell 1993, 73:1323-1337.

33. Pignoni F, Zipursky SL: Induction of Drosophila eye development by decapentaplegic. Development 1997, 124:271-278.

34. Schubiger M, Carre C, Antoniewski C, Truman JW: Ligand-dependent derepression via EcR/USP acts as a gate to coordinate the differentiation of sensory neurons in the Drosophila wing. Development 2005, 132:5239-5248.

35. D'Avino PP, Thummel CS: Crooked legs encodes a family of zinc finger proteins required for leg morphogenesis and ecdysone-regulated gene expression during Drosophila metamorphosis. Development 1998, 125:1733-1745.

36. Mitchell N, Cranna N, Richardson H, Quinn L: The ecdysone-inducible zincfinger transcription factor Crol regulates Wg transcription and cell cycle progression in Drosophila. Development 2008, 135:2707-2716.

37. Froldi F, Ziosi M, Garoia F, Pession A, Grzeschik NA, Bellosta P, Strand D, Richardson HE, Pession A, Grifoni D: The lethal giant larvae tumour suppressor mutation requires $\mathrm{dMyc}$ oncoprotein to promote clonal malignancy. BMC Biol 2010, 8:33.

38. Lehner CF, O'Farrell PH: The roles of Drosophila cyclins a and B in mitotic control. Cell 1990, 61:535-547.

39. Buszczak M, Paterno S, Lighthouse D, Bachman J, Planck J, Owen S, Skora AD, Nystul TG, Ohlstein B, Allen A, Wilhelm JE, Murphy TD, Levis RW, Matunis E, Srivali N, Hoskins RA, Spradling AC: The Carnegie protein trap library: a versatile tool for Drosophila developmental studies. Genetics 2007, 175:1505-1531.

40. Whitfield WG, Gonzalez C, Sanchez-Herrero E, Glover DM: Transcripts of one of two Drosophila cyclin genes become localized in pole cells during embryogenesis. Nature 1989, 338:337-340.

41. Quinn LM, Herr A, McGarry TJ, Richardson H: The Drosophila Geminin homolog: roles for Geminin in limiting DNA replication, in anaphase and in neurogenesis. Genes Dev 2001, 15:2741-2754.

42. Reis T, Edgar BA: Negative regulation of dE2F1 by cyclin-dependent kinases controls cell cycle timing. Cell 2004, 117:253-264.

43. Aza-Blanc P, Kornberg TB: Ci: a complex transducer of the hedgehog signal. Trends Genet 1999, 15:458-462.

44. Von Ohlen T, Lessing D, Nusse R, Hooper JE: Hedgehog signaling regulates transcription through cubitus interruptus, a sequence-specific DNA binding protein. Proc Natl Acad Sci USA 1997, 94:2404-2409.

45. Von Ohlen T, Hooper JE: Hedgehog signaling regulates transcription through Gli/Ci binding sites in the wingless enhancer. Mech Dev 1997, 68:149-156.
46. Mandell JG, Barbas CF: Zinc finger tools: custom DNA-binding domains for transcription factors and nucleases. Nucleic Acids Res 2006, 34:W516-W523.

47. Schneiderman Jl, Goldstein S, Ahmad K: Perturbation analysis of heterochromatin-mediated gene silencing and somatic inheritance. PLoS Genet 2010, 6(9):e1001095

48. Herranz H, Milan M: Signalling molecules, growth regulators and cell cycle control in Drosophila. Cell Cycle 2008, 7(21):3335-3337.

49. Edgar BA, O'Farrell PH: The three postblastoderm cell cycles of Drosophila embryogenesis are regulated in G2 by string. Cell 1990, 62:469-480.

50. Edgar BA, Lehman DA, O'Farrell PH: Transcriptional regulation of string (cdc25): a link between developmental programming and the cell cycle. Development 1994, 120:3131-3143.

51. Kylsten P, Saint R: Imaginal tissues of Drosophila melanogaster exhibit different modes of cell proliferation control. Dev Biol 1997, 192:509-522.

52. Hayashi S: Drosophila imaginal development and the escargot gene. Tanpakushitsu Kakusan Koso 1996, 41:1095-1103.

53. Aranda A, Pascual A: Nuclear hormone receptors and gene expression. Physiol Rev 2001, 81:1269-1304

54. Martinez E, Givel F, Wahli W: A common ancestor DNA motif for invertebrate and vertebrate hormone response elements. EMBO J 1991, 10:263-268

55. Oberdorster E, Clay MA, Cottam DM, Wilmot FA, McLachlan JA, Milner MJ: Common phytochemicals are ecdysteroid agonists and antagonists: a possible evolutionary link between vertebrate and invertebrate steroid hormones. J Steroid Biochem Mol Biol 2001, 77:229-238.

56. Nilsson M, Dahlman-Wright K, Gustafsson JA: Nuclear receptors in disease: the oestrogen receptors. Essays Biochem 2004, 40:157-167.

57. Kouzmenko AP, Takeyama K, Ito S, Furutani T, Sawatsubashi S, Maki A, Suzuki E, Kawasaki Y, Akiyama T, Tabata T, Kato S: Wnt/beta-catenin and Estrogen signaling converge in vivo. J Biol Chem 2004, 279:40255-40258.

58. Klaus A, Birchmeier W: Wnt signalling and its impact on development and cancer. Nature reviews 2008, 8:387-398.

59. Kassis JA, Noll E, VanSickle EP, Odenwald WF, Perrimon N: Altering the insertional specificity of a Drosophila transposable element. Proc Natl Acad Sci USA 1992, 89:1919-1923.

60. Dietzl G, Chen D, Schnorrer F, Su KC, Barinova Y, Fellner M, Gasser B, Kinsey K, Oppel S, Scheiblauer S, Couto A, Marra V, Keleman K, Dickson BJ: A genome-wide transgenic RNAi library for conditional gene inactivation in Drosophila. Nature 2007, 448:151-156.

61. Mitchell NC, Johanson TM, Cranna NJ, Er ALJ, Richardson HE, Hannan RD, Quinn LM: Hfp inhibits Drosophila myc transcription and cell growth in a TFIIH/Hay-dependent manner. Development 2010, 137:2875-2884.

62. Haring M, Offermann S, Danker T, Horst I, Peterhansel C, Stam M: Chromatin immunoprecipitation: optimization, quantitative analysis and data normalization. Plant Methods 2007, 3:11

\section{doi:10.1186/1471-213X-13-28}

Cite this article as: Mitchell et al.: The Ecdysone receptor constrains wingless expression to pattern cell cycle across the Drosophila wing margin in a cyclin B-dependent manner. BMC Developmental Biology 2013 13:28.

\section{Submit your next manuscript to BioMed Central and take full advantage of:}

- Convenient online submission

- Thorough peer review

- No space constraints or color figure charges

- Immediate publication on acceptance

- Inclusion in PubMed, CAS, Scopus and Google Scholar

- Research which is freely available for redistribution 